University of Nebraska - Lincoln

DigitalCommons@University of Nebraska - Lincoln

Martin Centurion Publications

Research Papers in Physics and Astronomy

2012

\title{
Dispersion compensation for attosecond electron pulses
}

\author{
Peter Hansen \\ University of Nebraska-Lincoln \\ Cory Baumgarten \\ University of Nebraska-Lincoln, cbaumga3@gmail.com \\ Herman Batelaan \\ University of Nebraska - Lincoln, hbatelaan@unl.edu \\ Martin Centurion \\ University of Nebraska - Lincoln, martin.centurion@unl.edu
}

Follow this and additional works at: https://digitalcommons.unl.edu/physicscenturion

Part of the Physics Commons

Hansen, Peter; Baumgarten, Cory; Batelaan, Herman; and Centurion, Martin, "Dispersion compensation for attosecond electron pulses" (2012). Martin Centurion Publications. 15.

https://digitalcommons.unl.edu/physicscenturion/15

This Article is brought to you for free and open access by the Research Papers in Physics and Astronomy at DigitalCommons@University of Nebraska - Lincoln. It has been accepted for inclusion in Martin Centurion Publications by an authorized administrator of DigitalCommons@University of Nebraska - Lincoln. 


\title{
Dispersion compensation for attosecond electron pulses
}

\author{
Peter Hansen, Cory Baumgarten, Herman Batelaan, and Martin Centurion ${ }^{\text {a) }}$ \\ Department of Physics and Astronomy, University of Nebraska-Lincoln, Lincoln, Nebraska 68588, USA
}

(Received 28 June 2012; accepted 1 August 2012; published online 20 August 2012)

\begin{abstract}
We propose a device to compensate for the dispersion of attosecond electron pulses. The device uses only static electric and magnetic fields and therefore does not require synchronization to the pulsed electron source. Analogous to the well-known optical dispersion compensator, an electron dispersion compensator separates paths by energy in space. Magnetic fields are used as the dispersing element, while a Wien filter is used for compensation of the electron arrival times. We analyze a device with a size of centimeters, which can be applied to ultrafast electron diffraction and microscopy, and fundamental studies. C 2012 American Institute of Physics. [http://dx.doi.org/10.1063/1.4746271]
\end{abstract}

The control of ultrafast electron motion with laser light has led to the study and use of attosecond processes. ${ }^{1}$ The generation of EUV-pulses, ${ }^{2}$ the observation of nuclear dynamics in a molecule, ${ }^{3}$ and electron emission from metal nano-tips ${ }^{4}$ are a few examples of exciting recent developments in this area. Attosecond resolution can currently be attained but only with recollision or recombination processes. In these processes, an ultrashort laser pulse ionizes an atom or molecule. The electron first moves away after which the laser field changes direction and consequently the electron reverses direction to recollide with the parent ion. This implies that the electron "pulse" is not readily available to study arbitrary targets. Conversely, in ultrafast electron microscopy (UEM) and ultrafast electron diffraction (UED), the source and the target are spatially separated by centimeters or more, which allows a much more general category of targets to be investigated. UEM and UED have been applied to investigate the motion of atoms in solid targets and isolated molecules, and produce images with picometer spatial resolution and femtosecond or picosecond temporal resolution. $^{5-8}$

While the motion of atoms is on femtosecond time scales and longer, the time scale for changes in electron orbitals is on the order of attoseconds. Recent theoretical calculations have shown that attosecond electron pulses could be used to image the motion of electrons in atoms and molecules with attosecond temporal resolution and picometer spatial resolution. ${ }^{9,10}$ This would require attosecond electron pulses with keV energy to be delivered on a target. The two main factors limiting the duration of electron pulses are space charge effects (Coulomb repulsion in multi-electron pulses) and dispersion due to their initial energy spread. In order to reach attosecond duration, it will be necessary to use single-electron pulses to eliminate space charge effects.

Here, we present a method to compensate for the dispersion of single-electron attosecond pulses. In order to use single-electron attosecond pulses in laser-pump electron-probe experiments, the electron pulses need to be synchronized with the laser pulses, and preferably run at a high repetition rate (on the order of $\mathrm{MHz}$ ) to accumulate sufficient counts for diffraction and imaging. Our proposed method satisfies both of

\footnotetext{
${ }^{\text {a) }}$ Author to whom correspondence should be addressed. Electronic mail: mcenturion2@unl.edu.
}

these conditions. Electrons can be extracted from a tip using a femtosecond laser oscillator at $\mathrm{MHz}$ repetition rates ${ }^{11,12}$ which automatically implies their synchronization. The synchronization is preserved because only static fields are used for dispersion compensation.

Other proposals to compress electron pulses either use high intensity lasers which are not compatible with high repetition rates ${ }^{13,14}$ or radio-frequency cavities that are currently limited by synchronization to the hundred-femtosecond regime. ${ }^{15,16}$ An advantage of time-dependent techniques is their capability to increase the energy spread of the pulse and thus reduce the minimum achievable pulse duration. The minimum pulse duration depends on the energy spread, as stated in the Heisenberg uncertainty principle. The electron dispersion compensator (EDC) proposed here does not change the energy spread and therefore can only recompress an electron pulse back to its original pulse duration. With the EDC, the original pulse duration at the source can be reproduced at the target position. An important advantage of the EDC is that it uses only static fields and therefore avoids the need to have synchronization on attosecond time scales. Existing pulse compression techniques for accelerator-based relativistic electron bunches include $\alpha$-magnets ${ }^{17}$ and chicanes, ${ }^{18,19}$ usually working in conjunction with RF-cavity electron sources. ${ }^{20}$ These time-independent compression techniques have reached the femtosecond domain. Additionally, chicanes have longer flight times for faster electrons as compared to slower electrons. This flight time dispersion can be used for RF-cavity sources, for which the fast electrons can trail the slow electrons. However, such an arrangement does not work for non-relativistic electron pulses generated from photo-cathodes or field emission tip sources, for which the slow electrons trail the fast electrons. For $\alpha$-magnets, it is interesting to note that the flight time dispersion changes sign between relativistic and non-relativistic velocities. However, just as chicanes the flight time dispersion has the incorrect sign at non-relativistic velocities.

A strong motivation for the EDC is the recent development of pulsed electron sources with ever shorter pulse durations: pulse durations below $100 \mathrm{fs},{ }^{21}$ sub-laser cycle durations (less than $2.7 \mathrm{fs}$ at a wavelength of $800 \mathrm{~nm}$ ), ${ }^{11}$ and even attosecond durations have been reported. ${ }^{4}$ Attosecond electron pulses disperse very rapidly due to an initial energy spread on the order of a few $\mathrm{eV}$, so in general it is not 
possible to place the target sufficiently close to the source to achieve attosecond resolution. For example, for a transform limited electron pulse with duration of 110 as (energy spread of $3 \mathrm{eV}$ ) and kinetic energy of $3 \mathrm{keV}$, the pulse will disperse at a rate of $16 \mathrm{fs} / \mathrm{mm}$.

We borrow an idea from optics, an optical dispersion compensator, and propose to use magnetic fields and a Wien filter (WF) to construct an electron dispersion compensator (Figure 1). Magnetic fields are used to spatially separate electrons with different kinetic energies, while the WF introduces a position-dependent delay. The first magnetic field points perpendicular to the plane of motion and turns the electrons with a radius proportional to their velocity. A second magnetic field of opposite sign turns the electrons back around, resulting in the same parallel paths, spatially separated by velocity. A balanced WF is used to introduce a velocity dependent time delay. A Wien filter employs crossed magnetic and electric fields that are balanced $\left(E=v_{0} B\right)$ so that an electron with speed $v_{0}$ experiences no net deflection force. When entering the Wien filter, the electron experiences an electric potential that depends on position (Figure 1). Passage through the WF leaves the electron velocity unchanged but introduces a time delay that is linearly dependent on the velocity of the electron. Two magnetic fields after the WF will spatially recombine the electron pulse. For an appropriate setting of the fields, the higher energy electrons can be delayed in such a way that they will catch up to the slower ones, recreating on the target the short pulse that was generated at the source.

An analysis based on classical trajectories is made. The first part is analytical and sets the physical parameters, while the second part is numerical, includes both velocity spread and angular spread of the initial electron distribution, and is used to assess the performance and function of the EDC. Additionally, an analysis of fringe fields is made. A classical approach is used. Care is taken that the calculated and reported widths do not approach the Heisenberg uncertainty limit, for example, the initial electron beam disperses at $16 \mathrm{fs} / \mathrm{mm}$. This leads to a temporal spread of

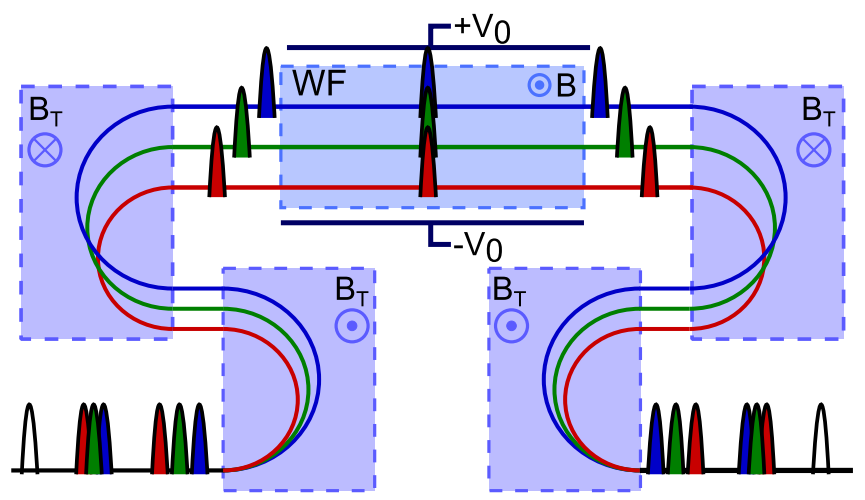

FIG. 1. Proposed setup for the EDC. An electron pulse is generated and disperses (left bottom). Subsequently, the electrons pass through two uniform magnetic fields $\left(\mathrm{B}_{\mathrm{T}}\right)$ and are spatially dispersed according to their kinetic energy. Higher (lower) energy electrons are indicated in blue (red). Green represents the trajectory of average energy electrons. Upon passing through a WF, the relative delay between the electrons is compensated. The WF is operated in its balanced mode where deflection due to parallel plates held at a potential $\mathrm{V}_{0}$ is compensated by deflection due to a magnetic field $\mathrm{B}$. The electrons are spatially recombined by two further magnetic field sections. approximately $5 \times 10^{2}$ fs at the entrance of the first deflection magnet, and a spatial spread of $1.5 \mu \mathrm{m}$. This far exceeds the coherence length of $v_{0} \Delta t=\hbar v_{0} / \Delta E \approx 6 \mathrm{~nm}$, and $\Delta x \Delta p \approx 2 \times 10^{-32} \gg \hbar$.

The following assumptions are made in the analysis. The electrons are non-relativistic, and the initial pulse is composed of a single electron. The velocity spread $\Delta v$ in the initial electron pulse is small with respect to the average velocity, $v_{0}$. For the calculations in the paper, we will use an energy spread of $3 \mathrm{eV}$ and kinetic energy of $3 \mathrm{keV}$, giving $\Delta E / E=10^{-3}$. For an initial energy spread of $3 \mathrm{eV}$, the minimum pulse duration allowed by the Heisenberg uncertainty principle is 110 as. The delay caused by the fringe fields around the Wien Filter is negligible with respect to the delay inside the filter. The electron source is assumed to be pointlike, motivated by the fact that the source size for a field emission tip is much smaller than any of the displacements calculated. We will first consider the velocity spread and second the additional angular spread of velocities at the source.

We start by considering an electron trajectory travelling parallel to the $\mathrm{x}$-axis (Fig. 1). After passing two magnetic field segments, the electron's trajectory is again parallel to the $\mathrm{x}$-axis. The separation $\Delta y$ between two trajectories that correspond to electrons with initial velocities different by $\Delta v$ is

$$
\Delta y=4 m \Delta v /\left(e B_{T}\right)
$$

where $m$ is the mass of the electron, $e$ is the elementary charge, and $B_{T}$ is the magnitude of the turning magnetic fields. The electric field in the WF is created by two parallel plates a distance $h$ apart in the $y$-direction and held at voltages $\pm \mathrm{V}_{0}$. The group velocity $v_{g}$ of an electron pulse after entering the Wien filter at a distance $\Delta y$ from the center, where the potential value is $\Delta V$, follows from energy conservation and is given by, 22

$$
v_{g}=v_{0} \pm \Delta v_{g}=v_{0} \pm \Delta V \sqrt{\frac{e}{8 m U_{a}}},
$$

where $\Delta V=2 V \Delta y / h, \Delta v_{g}$ is the change in group velocity, and $U_{a}$ is the potential used at the electron source to accelerate the electrons to their average kinetic energy, $\frac{1}{2} m v_{0}^{2}$. Now, we can write the change in velocity using the electric field $E=V / h$ and kinetic potential $U_{a}=m v_{0}^{2} / 2 e$ as

$$
\Delta v_{g}=\frac{e E \Delta y}{m v_{0}}
$$

Equations (1) and (3) can be combined to find the velocity change of an electron upon entrance into the Wien filter with an initial velocity $v_{0}+\Delta v$ to be

$$
\Delta v_{g}=\frac{4 E}{v_{0} B_{T}} \Delta v .
$$

The travel time through the WF is $T_{W F}=d / v_{g}$, where $d$ is the length of the filter. The relative delay $\Delta T_{W F}$ is given by

$$
\Delta T_{W F}=\frac{d}{v_{0}^{2}} \Delta v_{g}=\frac{4 E d}{B_{T} v_{0}^{3}} \Delta v .
$$


Now, we can compare the relative delay in the WF to the pulse duration at the target that is due to the initial velocity spread. The pulse duration $\sigma_{T}$ as a function of time is given by

$$
\sigma_{T}(t)=\frac{\Delta v}{v_{0}} t
$$

The dispersion in the magnetic fields can be ignored since electrons travelling with different velocities still have an equal transit time. For compensation to be effective, the relative delay (Eq. (5)) must be opposite to the pulse duration without compensation at the target (Eq. (6))

$$
\Delta T_{W F}=-\sigma_{T}(t) .
$$

Letting the total displacement be $l=v_{0} t$ we can get constraints for the system,

$$
\frac{4 E}{B_{T} v_{0}}=-\frac{l}{d}
$$

Experimentally, we are free to choose the average velocity, $v_{0}$, the distance to the target, $l$, and the length of the WF, $d$. These choices constrain the ratio $E / B_{T}$ in the EDC. Under these conditions, the dispersion of the pulse is compensated by the WF. This means that electrons starting simultaneously at the source will arrive simultaneously at the target. An important factor not considered in this analytical treatment is the case of an initial velocity with components perpendicular to the x-direction, which we accounted for using a numerical simulation.

The simulation includes a component of the velocity in the $\mathrm{y}$-direction, but propagation in the z-direction is ignored. This can be justified by choosing magnetic fields parallel to the z-axis. In this case, an initial velocity chosen along the z-direction will increase the approximate travel time from $l / v_{0}$ to $v_{0}^{-1} \sqrt{l^{2}+\left(v_{z} t\right)^{2}}$. For an initial electron beam divergence of $1 \mathrm{mrad}$ and propagation length of $10 \mathrm{~cm}$, this leads to a spread of $1.5 \mathrm{fs}$. However, an electron beam collimation at the source of $0.1 \mathrm{mrad}$ reduces the spread to 15 as. We proceed to analyze the effect of an angle spread on the y-direction.

We have developed a Fortran code to simulate the propagation by solving Newton's equations of motion $\partial^{2} \vec{r} / \partial t^{2}$ $=e / m(\partial \vec{r} / \partial t \times \vec{B}+\vec{E})$ with a Runge-Kutta-Verner fifthorder method. For each trajectory, the starting position is the origin, and the initial velocity in the $x-y$ plane is set to a specific angle and magnitude. We chose an angle spread of $1 \mathrm{mrad}$, and an energy spread of $\Delta E / E=10^{-3}$. The average kinetic energy of $3 \mathrm{keV}$ corresponds to an initial speed, $v_{0}$, of $3.23 \times 10^{7} \mathrm{~m} / \mathrm{s}$. The deflection fields are chosen at $3.1 \mathrm{mT}$ yielding a trajectory radius of $5.92 \mathrm{~cm}$. This sets a reasonable length scale for experiments. The target is displaced $10 \mathrm{~cm}$ from the source along the $\mathrm{x}$-axis. When the electron crosses the boundary at the beginning and at the end of the WF, the velocity in the $\mathrm{x}$-direction is set to

$$
v_{x f}=\sqrt{v_{x i}^{2} \pm \frac{2 e E}{m} \Delta y},
$$

where, $v_{x i}$, is the velocity in the $\mathrm{x}$-direction before the boundary, and the \pm sign indicates the electron entering or leaving the filter.
We have found that in order to achieve a sub-fs pulse duration, a non-uniform magnetic field must be used inside the WF. If the WF magnetic field strength $B_{W F}$ is set to $E / v_{0}$ then the Lorentz force is balanced for the electrons that have velocity $v_{0}$ and enter at the center of the filter. Other electrons will have a slight deflection exiting the filter, which is amplified by the remaining two magnetic fields. The assumption that the turning magnetic fields do not contribute any time delay holds only for electrons with velocity parallel to the $\mathrm{x}$-axis. This can be corrected by tuning $B_{W F}$ to match the velocity based on the vertical position of the electron. That is,

$$
B_{W F}(y)=\frac{E}{v_{W F}(y)},
$$

where $v_{W F}$ is the velocity of an electron in the WF. If the electron velocity is initially parallel to the $\mathrm{x}$-axis, then, using Eqs. (2), (3), and (8) we can write Eq. (10) as

$$
\begin{gathered}
B_{W F}=\frac{E}{v_{0}}(1-\varepsilon) \\
B_{W F}=\frac{E}{v_{0}}\left(1-\left(1-\frac{l}{d}\right) \frac{e B_{T}}{4 m v_{0}} \Delta y\right),
\end{gathered}
$$

using a first order Taylor expansion in the small parameter $\varepsilon$. The tuning of $B_{W F}$ compensates for electrons entering the WF with different velocities, but introduces complications to the analytical solution for electrons entering the WF at an angle. When an electron enters the Wien filter at an angle, its trajectory deviates from a straight line. This along with the $\mathrm{y}$-dependence of the magnetic field leads to a differential equation of motion that has no analytic solution.

Figure 2 shows the numerical results for an initial pulse with an angle spread $\Delta \theta$ of $1 \mathrm{mrad}$ and energy spread $\Delta E / E$ of $10^{-3}$. The minimum pulse duration was found at the designed target distance of $10 \mathrm{~cm}$. The resulting pulse width at the target is $1.9 \times 10^{-3} \mu \mathrm{m}$ which corresponds to a pulse duration of 60 as. The initial pulse duration in this simulation was zero, thus the resulting pulse duration indicates the spread in arrival times. Assuming a classical Gaussian initial pulse width of 110 as duration and adding the spread in

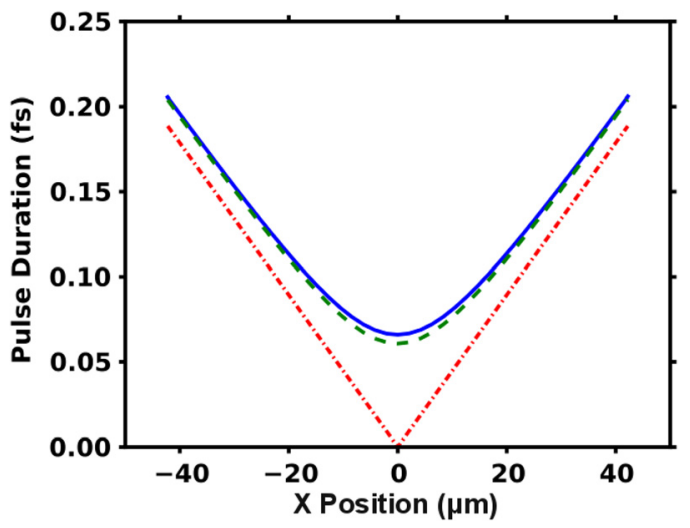

FIG. 2. Pulse duration at the target position. The duration of the pulse is given as a function of the target position, $\mathrm{X}$. The dash-dotted (red) line indicates the analytic solution assuming straight trajectories through the WF and no angular spread. The dashed (green) line indicates the numerical result including an energy spread, while the solid (blue) line indicates the numerical result that includes both the energy and angular spread. 


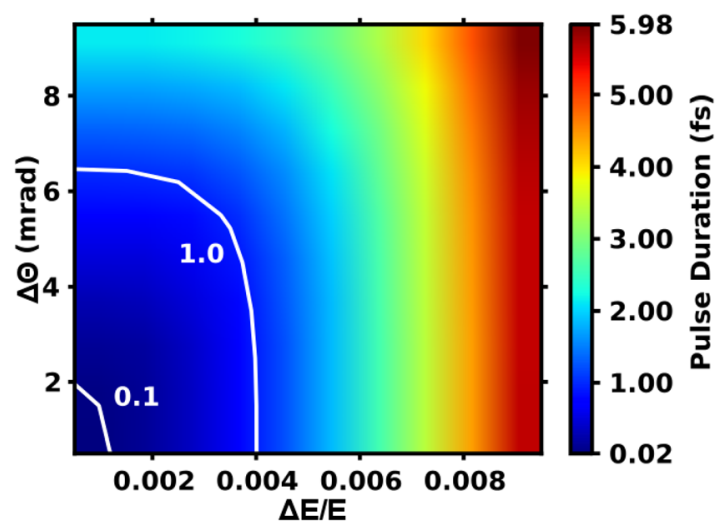

FIG. 3. Optimal focus. The minimum focus (in femtoseconds) is given as a function of initial energy spread and angular spread. The white contours correspond to the areas where the temporal spreads are below $0.1 \mathrm{fs}$ and $1.0 \mathrm{fs}$.

quadrature gives a pulse length at the target of 125 as. Adding this initial pulse duration which is consistent with the Heisenberg uncertainty principle results in pulse durations that exceed the quantum limit at all times. Our simulations show that the spread of the pulse duration is below 200 as over a length of $80 \mu \mathrm{m}$, a sufficient length to interact with either a solid or a gaseous target. Furthermore, this focus is achieved at a distance of $10 \mathrm{~cm}$ from the source, a practical distance for most applications.

Figure 3 shows the minimum pulse duration at the target as a function of the initial angle spread and energy spread. The smaller contour corresponds to the area where the spread in the pulse duration is below $0.1 \mathrm{fs}$. The larger contour shows the area where the pulse duration is 1.0 fs or less. The pulse duration is significantly shorter than one femtosecond even if the energy spread is increased to $3 \times 10^{-3}$ and the angle spread is increased to $3 \mathrm{mrad}$. The device is robust to variation of energy and angle spread over a fairly wide range. We have also verified that the position of the temporal focus changes linearly with the potential of the Wien filter (for changes limited to one percent). The duration of the pulse at the focus is not affected by the potential. In practice, this means that a relative accuracy and stability of $8 \times 10^{-4}$ of the balancing electric field of $10^{5} \mathrm{~V} / \mathrm{m}$ leads to changes of the focal length of $80 \mu \mathrm{m}$, which in turns keeps the pulse duration to within 200 as. This relative accuracy and stability is readily attained with off-the-shelf electronics.

Several additional tests were performed to validate the simulation results. The resulting pulse width converges for decreasing time steps and energy conservation is tested at each step. A backward propagation is performed by reversing the velocities at the end of the trajectory and propagating the electrons back through the EDC for the same amount of time. We estimate the numerical error of the spread to be 75 as. We have also simulated the motion of an electron passing through the fringe fields of the WF, which showed that the delay remains linear as a function of entrance position with respect to the Wien filter. The propagation of the electrons was simulated using the General Particle Tracer (GPT), a commercially available software package. ${ }^{23}$ The fringe fields of the Wien filter were calculated for two parallel plates in a 2D geometry using Poisson Superfish. ${ }^{24}$
In conclusion, we have shown a dispersion compensator for attosecond single-electron pulses that uses only static magnetic and electric fields. For multi-electron pulses, we do not expect that the device can achieve attosecond pulse durations, because of electron-electron interaction. The device is ideal to deliver ultrashort electron pulses synchronized to a laser pulse. The pulse duration on target can be as short as the pulse duration at the source, within hundreds of attoseconds. We have demonstrated this analytically for the simpler case where the initial velocity is longitudinal, and numerically for the case where there is a spread in both the magnitude and direction of the velocity. The duration of the pulses at the target could be measured using ponderomotive scattering from a short laser pulse, ${ }^{14,25}$ or by diffracting from a fast changing atomic or molecular target. ${ }^{10}$

We gratefully acknowledge support by the National Science Foundation (NSF) under Grant No. 0969506, and by the Department of Energy, Office of Science, Basic Energy Sciences, under Grant No. DE-SC0003931.

${ }^{1}$ P. B. Corkum and F. Krausz, Nat. Phys. 3, 381 (2007).

${ }^{2}$ E. Goulielmakis, M. Schultze, M. Hofstetter, V. S. Yakovlev, J. Gagnon, M. Uiberacker, A. L. Aquila, E. M. Gullikson, D. T. Attwood, R. Kienberger, F. Krausz, and U. Kleineberg, Science 320, 1614 (2008).

${ }^{3}$ S. Baker, J. S. Robinson, C. A. Haworth, H. Teng, R. A. Smith, C. C. Chirilă, M. Lein, J. W. G. Tisch, and J. P. Marangos, Science 312, 424 (2006).

${ }^{4}$ M. Krüger, M. Schenk, and P. Hommelhoff, Nature 475, 78 (2011).

${ }^{5}$ A. H. Zewail, J. Phys. Chem. A 104, 5660 (2000).

${ }^{6}$ A. H. Zewail, Annu. Rev. Phys. Chem. 57, 65 (2006).

${ }^{7}$ P. Reckenthaeler, M. Centurion, W. Fuß, S. A. Trushin, F. Krausz, and E. Fill, Phys. Rev. Lett. 102, 213001 (2009).

${ }^{8}$ R. J. D. Miller, R. Ernstorfer, M. Harb, M. Gao, C. T. Hebeisen, H. JeanRuel, C. Lu, G. Moriena, and G. Sciaini, Acta Crystallogr., Sect. A: Found. Crystallogr. 66, 137 (2010).

${ }^{9}$ P. Baum, J. Manz, and A. Schild, Sci. China, Ser. G 53, 987 (2010).

${ }^{10}$ H.-C. Shao and A. F. Starace, Phys. Rev. Lett. 105, 263201 (2010).

${ }^{11}$ P. Hommelhoff, C. Kealhofer, and M. A. Kasevich, Phys. Rev. Lett. 97, 247402 (2006).

${ }^{12}$ S. A. Hilbert, A. Neukirch, C. J. G. J. Uiterwaal, and H. Batelaan, J. Phys. B 42, 141001 (2009).

${ }^{13}$ P. Baum and A. H. Zewail, Proc. Natl. Acad. Sci. U.S.A. 103, 16105 (2006).

${ }^{14}$ S. A. Hilbert, C. Uiterwaal, B. Barwick, H. Batelaan, and A. H. Zewail, Proc. Natl. Acad. Sci. U.S.A. 106, 10558 (2009).

${ }^{15}$ L. Veisz, G. Kurkin, K. Chernov, V. Tarnetsky, A. Apolonski, F. Krausz, and E. Fill, New J. Phys. 9, 451 (2007).

${ }^{16}$ T. van Oudheusden, P. L. E. M. Pasmans, S. B. van der Geer, M. J. de Loos, M. J. van der Wiel, and O. J. Luiten, Phys. Rev. Lett. 105, 26 (2010).

${ }^{17}$ P. Kung, H. C. Lihn, H. Wiedemann, and D. Bocek, Phys. Rev. Lett. 73, 967 (1994).

${ }^{18}$ S. G. Anderson, J. B. Rosenzweig, P. Musumeci, and M. C. Thompson, Phys. Rev. Lett. 91, 074803 (2003).

${ }^{19}$ D. H. Dowell, J. L. Adamski, T. D. Hayward, P. E. Johnson, C. D. Parazzoli, and A. M. Vetter, Nucl. Instrum. Methods Phys. Res. A. 393, 184 (1997).

${ }^{20}$ For a review see: W. E. King, M. R. Armstrong, O. Bostanjoglo, and B. W. Reed, "High-speed electron microscopy," in Science of Microscopy, (Springer, 2007), Vol. I, pp. 406-444.

${ }^{21}$ B. Barwick, C. Corder, J. Strohaber, N. Chandler-Smith, C. Uiterwaal, and H. Batelaan, New J. Phys. 9, 142 (2007).

${ }^{22}$ M. Nicklaus and F. Hasselbach, Phys. Rev. A 48, 152 (1993).

${ }^{23}$ Pulsar Physics, Burghstraat 47, 5614 BC Eindhoven, The Netherlands.

${ }^{24}$ Los Alamos National Laboratory, LAACG, Los Alamos Accelerator Code Group. (See documentation LA-UR-96-1834).

${ }^{25}$ C. T. Hebeisen, G. Sciaini, M. Harb, R. Ernstorfer, T. Dartigalongue, S. G. Kruglik, and R. J. D. Miller, Opt. Express 16, 3334 (2008). 